INTERNATIONAL DESIGN CONFERENCE - DESIGN 2018

https://doi.org/10.21278/idc.2018.0291

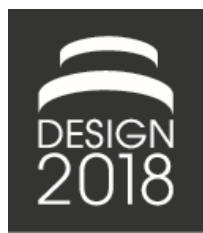

\title{
AESTHETIC PRODUCT INTERACTION: THE NECESSITY OF CONSISTENCY BETWEEN FUNCTION \& EMOTION
}

\author{
K. Lee, J. A. Self and H. Hong
}

\begin{abstract}
We investigate the application of aesthetic stimuli's influence on emotional response during product interaction; implications for novel yet acceptable product experiences. Adopting a research-throughdesign approach, three tape-dispenser products were designed. Together with a control, the dispensers offered visual and auditory stimulation during product use. Results indicate the importance of consistency between function and stimulus for elicitation of positive response towards novel aesthetic interventions. User profiles also appeared to implicate response to aesthetic stimuli.
\end{abstract}

Keywords: design research, innovation, interaction design

\section{Introduction}

The current study explores the tension between novelty and acceptability in innovative product design (Hekkert et al., 2003; Hung and Chen, 2012). In particular we examine how the application of aesthetics aimed at the stimulation of visceral response leading to emotional arousal (Hallnas, 2011; Bergghman and Hekkert, 2016) may provide opportunities for novel product experiences. In doing we identify product innovation as different from product invention (Rampino, 2011). Invention merely focuses on a result of discovering and creating products or introducing processes for the first time. The positive result received by a new product or process is not considered. Product innovation, on the other hand, occurs when a product achieves a significant contribution leading to the creation of different product meanings (Verganti, 2008).

However, in order to creative innovative products, it is necessary to add value through novel but appropriate product solutions, both in terms functionality and aesthetics. The current study explores how product innovation may be achieved through interventions aimed at radically changing product aesthetics (both visual and auditory) to introduce novel and unexpected product experiences. In this we explore the extent to which novel product aesthetics may influence initial emotional response and implications for the user's holistic product experience (Norman, 2004; Bergghman and Hekkert, 2016). Building upon the existing literature in this growing field of study (Norman, 2004; Desmet and Hekkert, 2007; Demir et al., 2009), we conclude with a discussion of relations between novel product aesthetics as driver for innovative meaning change (Verganti, 2008), emotional response and the holistic product experience.

\section{Product novelty and meaning innovation}

As interest in innovation has increased, design researchers have likewise begun to explore design as driver for innovative product development. Representatively, Rampino (2011) describes design-driven innovation through a hierarchical pyramid model. Three levers, as drivers for innovation, are positioned 
as: form, technology and mode-of-use, influencing both the form and function of the product. The use of these three levers, or their use concurrently, may result in both incremental and radical changes. For the purposes of our current study, Rampino's (ibid) form and mode-of-use levers are interesting in their positioning of, on the one hand, aesthetics, and the other, function as potential drivers towards innovative product solutions. However, departing from Rampino's (ibid) conceptual division of aesthetic and function as different paths towards product innovation, the current study suggests how careful consideration of their synthesis can provide the best opportunity for novel, yet functional product experiences (Norman, 2002).

As a result of the designers use of the three innovation levers Rampino (op cit) goes on to describe four categories of potential outcome: aesthetic, mode-of-use, meaning and finally typological innovations. Verganti (2008) further describes types of innovation according to degree of change in product meaning. When incremental changes of meaning occur, these lead to market-pull innovation to satisfy users and better reflect their needs. On the other hand, radical change of meaning can lead to design-driven innovation, with the potential for increasingly novel product expereinces. Both Rampino (2011) and Verganti $(2008,2009)$ emphasize the importance of meaning change in design innovation. For example, innovation that takes place simply through user-centred design does not often change meaning. However, innovation derived from radical change of meaning can lead to paradgm shift in our understanding of what a particular product is and should be; or radical meaning innovations. For example, the Italian Kitchenware producers Alessi (Verganti, 2003) radically changed the meaning of kitchen products from appliances and tools to playful objects through radical changes in aesthetic. This change then provided opportunities for the re-interpretation of kitchen products from purely utilitarian tools to objects of desire (i.e. Alessi's Form Follows Fiction kitchen range). Alessi's innovative kitchen products not only retained the functionality of kitchen utensil, but also drew upon the role of emotion in user/product experiences to provide new meanings.

Desmet and Hekkert (2007) further defines interaction between user and product as the product experience, which includes aesthetic experience, or the degree to which our senses are gratified, the meanings we attach to the product experience and the emotional experience that we feel in response to sensorial stimulation. In particular, Desmet and Hekkert (op cit.) states that the emotional experience during product interaction represents what the user feels towards the product; if it is beneficial and if to keep using the product for example (Desmet and Schifferstein, 2012). In addition, the emotions that the user experiences during product use have implications for other aspects, such as user satisfaction, attachment to the product, as well as rationales towards product purchase (op cit).

\section{Product aesthetics}

One other factor that greatly affects the user's emotional response during the user/product experience, and one that can potentially provide opportunities for novelty and meaning change, is the product's perceived aesthetic beauty. Thus, designers attempt to embed product value through the provision of aesthetic improvements (Locher et al., 2010). However, the concept of aesthetic has gradually begun to change. Researchers have moved beyond focusing on enhancing external beauty, to describe the product experience as a more holistic, emotional event including others sensorial experiences derived from both haptic and auditory interactions. This change has resulted in a more holistic interpretation of the aesthetics of the user/product experience.

In this respect Tractinsky (2005) defines interactive systems, as an aesthetic interaction that employs a variety of senses triggering an emotional response towards the product. Ross (2010) further explores the principles that underpin aesthetic product interaction, to suggest the ways in which it may elicit differing product/user experiences (Udsen and Jørgensen, 2005). Towards this effort, Desmet et al. (2008) introduced five elements as potential causes of aesthetic interaction: force, sound, motion, texture and performance. Together these five constructs are positioned to describe the subjective product attributes that contribute to a dominant versus an elegant interaction personality. Drawing upon these works highlighting the importance of a variety of sensory stimulation; implications for the product experience, the current study isolates sound and visual elements in an attempt to better understand their influence as drivers for more novel product experiences in pursuit of Verganti's (2008) notion of changed product meanings leading to radical product innovation. 
Adopting Desmet et al.'s (2008) notion of aesthetic interaction, the current study explores how novel visceral stimuli (Norman, 2004) may influence initial emotional response and implications for the ascription of meaning during the product experience. Here we focus on what Norman (ibid) describes as the initial visceral response to the product experience, and implications for the arousal of particular emotions. These visceral responses can be described as related to sensorial engagement (a smell, feel, sound), which then translates into an emotional response (i.e. curiosity, excitement, disinterest). Here we focus upon the initial product experience (first time product use), and implications for emotional response, rather than an analysis of the product experience over time. Thus, product experience is defined here as the user's engagement with and emotional response to the products application for the purposes of achieving a functional task (i.e. the use of a kitchen tool to achieve a cooking related objective).

\section{Methods}

The aim of the current study was to identify how visceral stimulus (Norman, 2004) may affect users' aesthetic response during the product experience. To then consider results in terms implications for stimulation of meaning change through novel aesthetic experiences as driver for innovation (Verganti, 2008). To achieve this research aim we applied novel stimuli to a well-known product (tape-dispenser). Tape dispenser was chosen as product type due to its ubiquity, its focus on practicality and the fact that existing re-designs from the original 3M design (American Chemical Society, 2017) have focused upon incremental changes to product form as driver for differentiation (Figure 1). As such, a tape-dispenser was seen as a product with which participants would be familiar, thus providing opportunity for the visceral stimulation to appear particularly novel. Novelty was important, not only because of our interest in meaning innovation (Verganti, 2009), but to attempt to explore any relationship between novel stimulation and emotional response. Adopting a mixed methods approach, degree of novelty was explored through semi-structured participant interviews, while data related to emotional response was gathered through the self-report PrEmo (SusanGroup, 2017) tool.
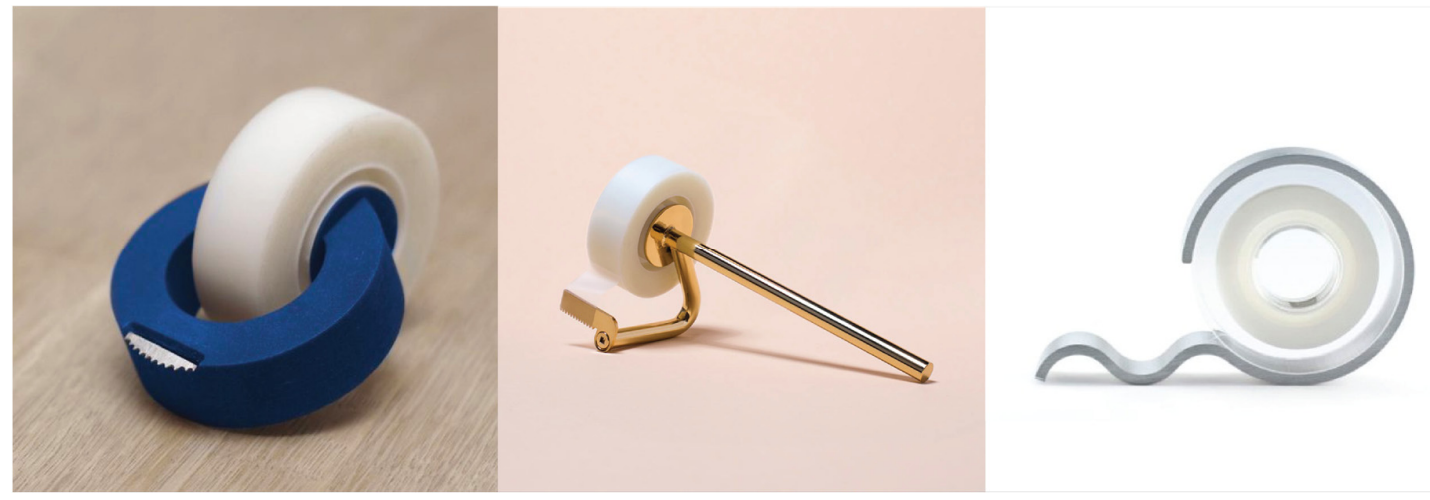

Figure 1. Existing redesigned tape dispensers only focus on appearance aesthetic; designed by Derk Reilink, 2017 (left), M-S-D-S Studio, 2015 (centre) and Mamoru Yasukuni, 2011 (right)

\subsection{Design and prototyping}

Adopting a research-through-design methodology (Frens, 2007) we examined previous approaches to the redesign of tape dispensers (Figure 1) and identified a focus upon aesthetic change. However, this approach cannot lead to design-driven innovation. As noted by Rampino (2011), innovation in design should change the meaning of a product. Therefore, a starting point for our re-design was to provide radical change in the aesthetic product experience as means to stimulate emotional response and meaning change.

Adopting the research-through-design approach (see also Zimmerman et al., 2007) the authors designed, developed and prototyped three product designs, which were then used to gather data to understand how visceral stimuli (visual and auditory) may implicate emotional response during product interaction. To 
this end we designed and prototyped three tape dispensers, adding visceral stimuli to two of the redesigns, with a third used as control (Figures 2 and 3).

First, Type $A$, was designed to approximate a conventional tape dispenser. To be used as a control to compare the effects of the visceral stimuli embedded within Types $B$ and Type C (Figures 2 and 3).

We applied visual stimuli to Type $B$ through the prevision of a visually novel animation. Users were able to see the animation in the style of a flipbook as they pulled the tape through the dispensing hole in the dispenser (Figure 3). We attached the animation on to an axis of tape to rotate continuously as the tape was extracted.

In contrast to Type $B$ (visual stimuli) we applied auditory feedback to Type $C$, providing a music box effect playing the 'Happy Birthday' tune whenever the dispenser is in use. Gears were used to centre the tape and the music box axis so that the music played when the dispenser was in use. In order to focus our attention upon the ways in which the visceral stimuli affected emotional response during user/product interaction, we unified the forms of the three tape dispenses to control for the influence of other aesthetic dimensions (i.e. form, materials, colours, finish).

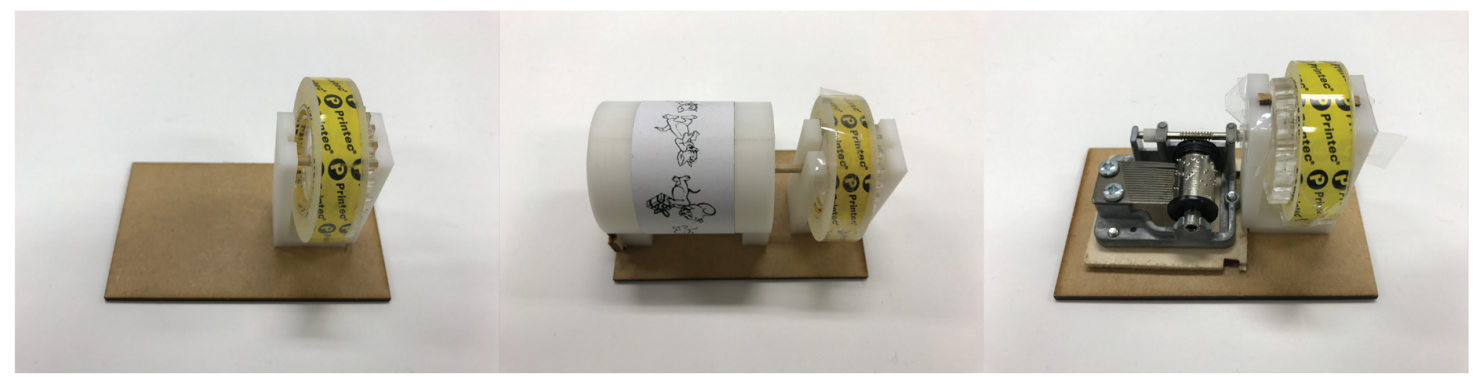

Figure 2. Redesigned tape dispenses inner part: Type A control (left), type B visual (centre) and type $\mathrm{C}$ auditory (right)

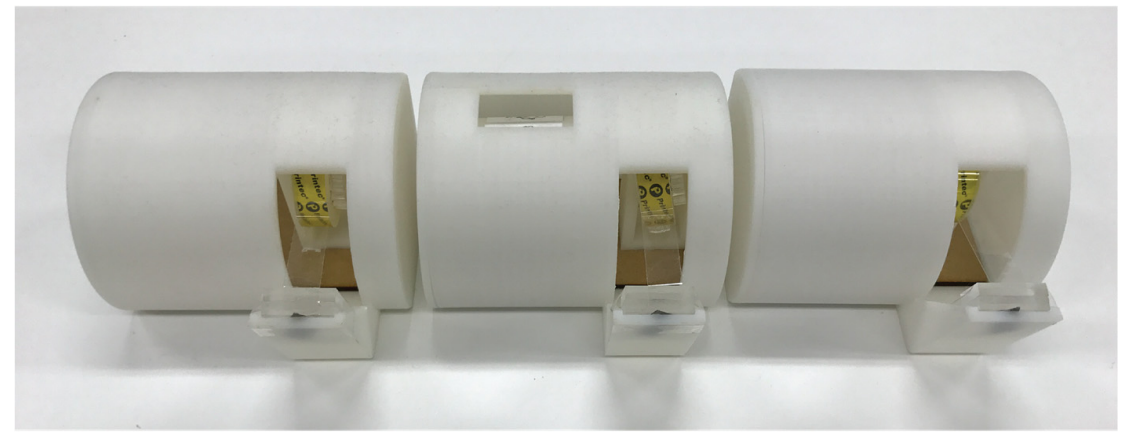

Figure 3. The redesigned tape dispensers' standardised form and casing design

\subsection{Participants}

The study was advertised to solicit participation from students studying at the authors' home institution in Korea. As a result 20 participants $(n=20)$ were selected through purposeful sampling (11 males and 9 females, with a mean age of 25.9 years).

\subsection{Empirical study procedure}

We prepared an experiment with the three prototype designs (Figures 2 and 3 ) to examine how the added aesthetic stimuli (animation, Type B visual and 'Happy Birthday' tune, Type C auditory) affected emotional response during the product/user experience. A task was prepared for participants to use the tape dispenser naturally and provide an emotionally assessable experience; participants were required to package gifts with wrapping paper. This activity provided a more natural experience when evaluating the three prototype stimuli. Thus, adopting both qualitative and quantitative approaches to data 
collection, the empirical study aimed to explore participants' responses to the three stimuli designs (Figures 4 and 5).

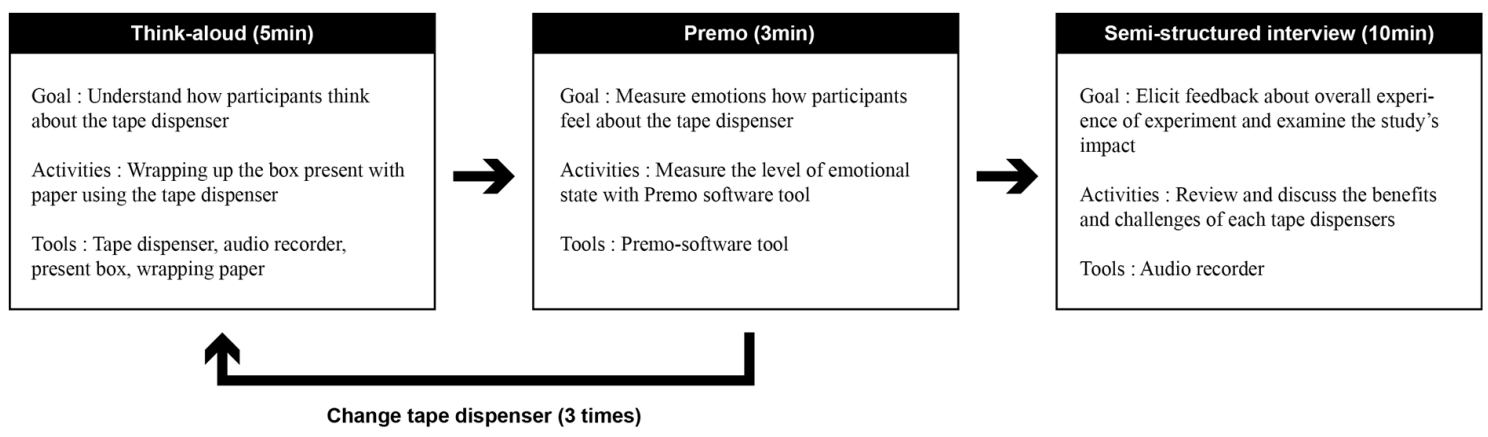

Figure 4. Empirical study procedure

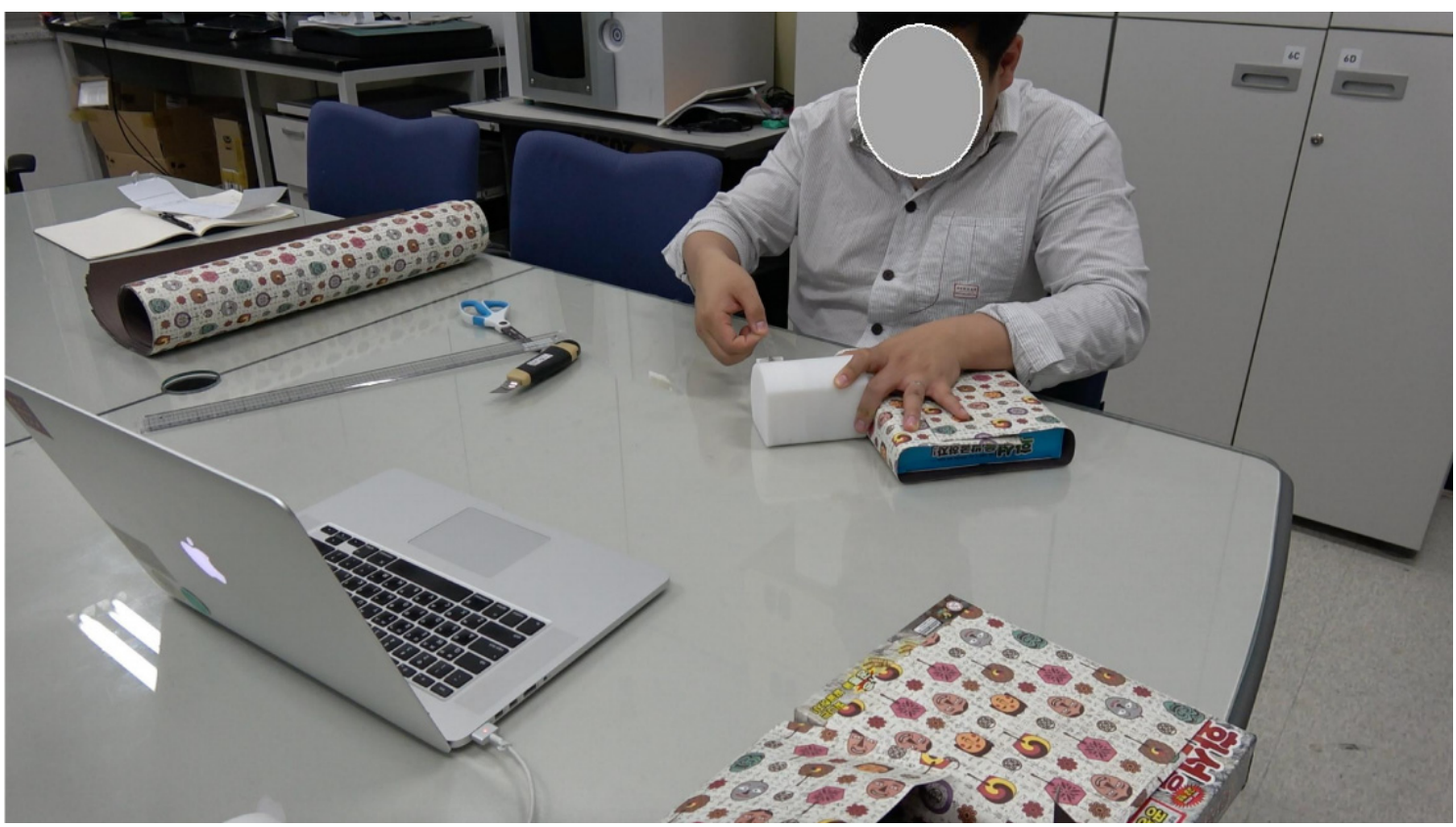

Figure 5. Gift wrapping study session in progress

First, participants were asked to wrap a gift box with paper using one of the three tape dispenser designs (randomised to limit order effect). During the wrapping process participants were encouraged to suggest what they thought of each of the tape dispenser designers (Think-aloud, Figure 4). The participants then recorded their emotional respone towards the dispenser designs through the PrEmo emotional response tool (SusanGroup, 2017). Adopting this approach we realise that the unwrapping experience is in itself highly emotive. However, with the inclusion of the base-line control design (Type A) we aimed to limit the influence of activity, as far as possible.

PrEmo, as a tool for collecting emotional response through animations and sounds based on 12 scaled response items (Figure 6), was selected as means to gather participant responses. The reason for the tool's selection was its potential to engage participants when compared to conventional self-report tools (i.e. survey response, Likert-scale etc.). The PrEmo tool offered greater potential to engage participants through its graphically animated user-interface design.

Applying the Premo tool, the 12 emotional response icons illustrated in Figure 6 were provided to participants. This resulted in 12 scaled (from 1 to 4) responses per participant. These self-report responses, derived from the PrEmo study, were then triangulated with open response data derived from semi-structured interview sessions and the Think-aloud study. 


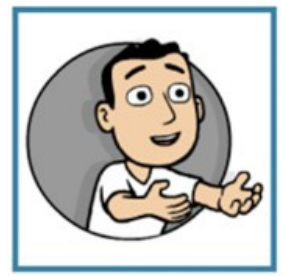

desire

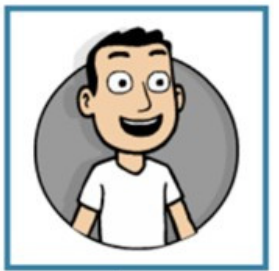

joy

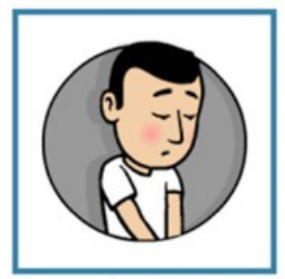

shame

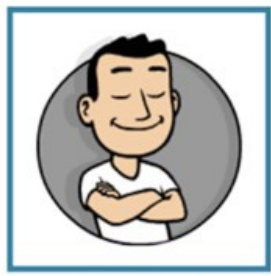

satisfaction

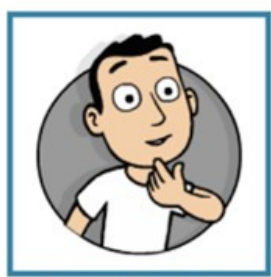

fascination

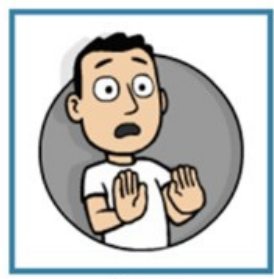

fear

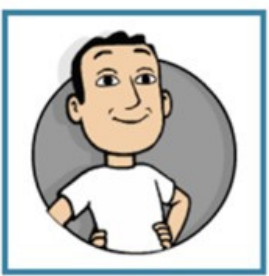

pride

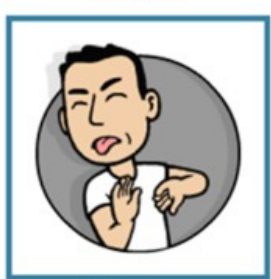

disgust

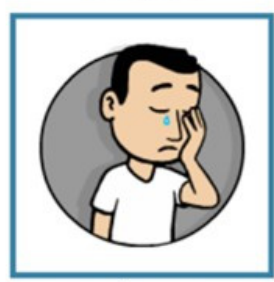

sadness

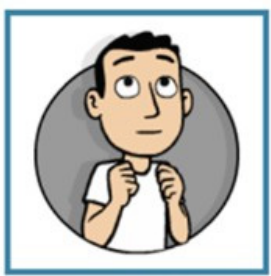

hope

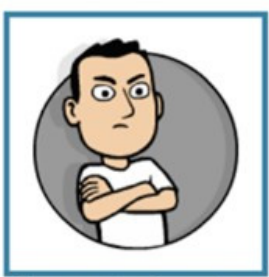

dissatisfaction

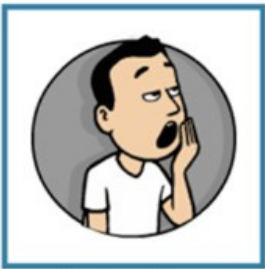

boredom

Figure 6. The PrEmo emotional response tool (SusanGroup, 2017)

Following the PrEmo and think-aloud sections of the study sessions we conducted semi-structured interviews to review and discuss the benefits and challenges of each tape dispenser design, eliciting qualitative feedback on the participants' overall product experience (see Figure 4).

\subsection{Analysis}

Interviews were recorded and subsequently transcribed. To ensure the anonymity of participants, we eliminated any identifiable information from the data-sets. We then analysed the data using a Grounded Theory approach (Strauss and Corbin, 1994). Through open encoding of the transcribed interviews, themes related to the identification of ideas within the transcribed interviews were organised into categories. This process continued until no new categories were identified. All transcribed interview data was then segmented through a thematic approach (identification of ideas that appeared to hang together) and classified through the application of identified categories. PrEmo responses were analysed descriptively through mean responses $(x)$ towards each of the 12 emotional response items, with results compared across the three dispenser designs.

\section{Results}

\subsection{General orientation}

Figure 7 illustrates participant responses towards each of the three dispenser designs: Type $A$ (control), or the standard dispenser design (no aesthetic interaction). Type $B$ (Visual); with a visual intervention, and Type $C$ (auditory), that provided the auditory change of a playful sound during product interaction ('Happy Birthday' tune). Figure 7's horizontal axis shows the 12 PreMo emotion response items, the vertical axis indicates mean $(x)$ responses towards each of the 12 response items. The green area of the graph (Figure 7, left) illustrates results for positive emotional response items (desire, satisfaction, pride, hope, joy, fascination, admiration), while the red area (right) indicates negative emotional response (disgust, dissatisfaction, shame, fear, sadness, boredom, contempt). Each tape-dispenser is represented by a different colour to aid clarity of comparison. 


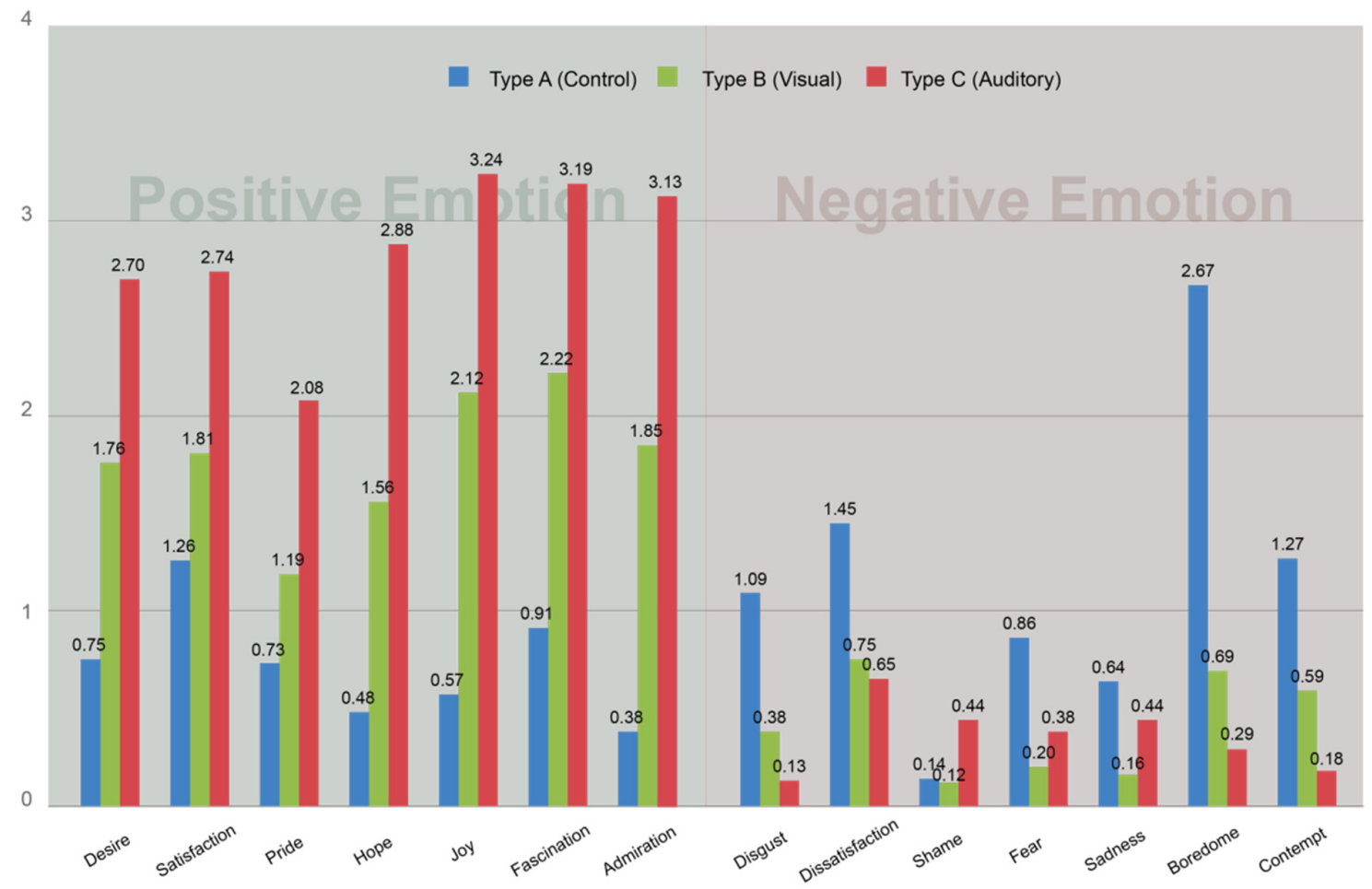

Figure 7. Descriptive PrEmo statistical responses across three tape dispenser types

As illustrated in Figure 7 the participants' recorded response towards each tape dispenser differed significantly. The most obvious result is that the Type $C$ (Auditory) design received increased positive responses (red bars, Figure 7, left) across all seven positive response items. Type $C$ (Auditory) also received the lowest mean $(x)$ response across 4 of the 7 negative response items (Disgust $x=0.13$, Dissatisfaction $x=0.65$, Boredom $x=0.29$, Contempt $x=0.18$ ).

In contrast Type $A$ (Control) received the lowest mean $(x)$ response across all 7 positive emotional response items (Figure 7, left, blue bars), and highest for 6 out of 7 negative emotional responses (Figure 7, right blue bars). In terms positive response, Type B (Visual) positioned between Type $C$ (Auditory) and Type $A$ (Control). For negative response items, the descriptive statistics showed Type $B$ (Visual) received mean responses $(x)$ between Type $A$ (Control) and Type $C$ (Auditory) for 4 of the 7 negative response items (Figure 7, Disgust $x=0.38$, Dissatisfaction $x=0.75$, Boredom $x=0.69$, Contempt $x=$ $0.59)$.

Holistically, this result indicated Type $C$ (Auditory) as providing the most positive product experience, followed by Type $B$ (Visual) and finally, Type $C$ (Control). This result was less surprising in that the embedded aesthetic stimuli (animation and 'Happy Birthday' tune) may have positively influenced the participants' gift wrapping experience. However, the following section further examines this result at a finer granularity of analysis, including qualitative participant response data from the think-aloud and interview components of the empirical study. We indicate relations between the potential emotional benefit of aesthetic product experiences and necessity to achieve functional product requirements (i.e. wrapping a gift); with potential implications for novel product experiences through aesthetic interventions.

\subsection{Comparison between designs}

The most notable difference in results between the three dispenser designs within the 7 negative response item was recorded for the Boredom response (Figure 7, Type $A x=2.67$, Type B $x=0.69$, Type $C x=0.29$ ). Together with the higher boredom response, mean $(x)$ responses across all 7 negative response items indicated the Control design (Type A) as stimulating the least positive user/product experience. This 
result was supported by the finding that Type $A$ (Control) also attracted reduced mean responses $(x)$ across all 7 positive response items (Figure 7, left, blue bars).

An analysis of interview data indicated the control's standard or expected design as reason for comparatively low positive and high negative participant responses. For example, Participant 07 discussed Type A as, "not different from an existing tape dispenser. I have no idea". In this response the participant appears to indicate surprise that Type A (Control) has no novel or unexpected features, and that this then may have confused the participant. This could also be a result of the experiment design, in that participants compared two unexpected designs (Type B Visual and Type C Auditory) with the standard control. However, other qualitative responses also provided evidence to suggest participant disinterest with Type $A$ (control design) as related to the design itself, rather than experimental conditions, "It is too boring. I could not feel anything different" (Participant 10). Taken together with descriptive statistical results (Figure 7) qualitative data indicated how the Type A (control) was seen as unexceptional when compared to the other two designs. This result is not unexpected in that the standard design failed to stimulate positive emotional response towards the product experience in comparison to the novel aesthetic interactions embedded within the other two dispenser designs.

More interesting, however, was qualitative response data indicated, although Type A (Control) was not successful in stimulating a more novel experience, the design was seen as competent in its ability to achieve the task of gift wrapping. For example, Participant 03 indicated, "It works well. It has no special features, but I'm satisfied with the function of cutting tape". Although the control did not attract increased positive response through the PrEmo self-report tool, qualitative results indicated participant satisfaction with its utilitarian focus. This result supported existing work related to product novelty and functionality, suggesting the critical balance between stimulation of novelty and acceptability founded upon functional expectations (Pahk and Self, 2015).

Related to the above, responses towards Type $B$ (Visual), indicated how embedding the playful animation within the dispenser served to stimulate interest, "The animation makes me feel curious" (Participant 07). Participant 15 also added, "I have not seen it before, it is so interesting". These qualitative responses thus indicated the potential reason for increased mean response across the 7 positive response items for Type B (Figure 7, left), when compared to Type A (Control). The novelty of the animation stimulated a more positive aesthetic product experience. This is again an expected result. More interesting was how the novelty of the animation also may have resulted in some confusion rooted in its interruption of the core task of wrapping a present, "It's good. However, I'm confused when I use this dispenser because it feels like I have to see both tape cutting and the animation" (Participant 16). Participant 8 also mentioned, "Type B is also a new approach, but it was hard to see it while cutting the tape. Type C, however, was easier to use and fun because it sounds natural".

Qualitative results indicated contradicting views between individual participants. For example, Participant 03 indicated, "I prefer Type B [Visual] rather than Type A [Control] because the tape dispenser so far was not funny and is only for the function of disconnecting the tape". Here Participant 03 appears most concerned with the aesthetic aspects of the design (animation) and its resulting novelty. In contrast Participant 08 stated, "I prefer Type A more than Type B because B is functionally difficult to use and strange for me". This result points to the particular profile of the user as implicating response to aesthetic product experiences. It also agrees with existing work on novelty in design to indicate a balance between a desire for difference and the necessity of fulfilling functional expectations (Bergghman and Hekkert, 2016).

Holistically, the visual aesthetic interaction appeared to stimulate positive emotion, but also cause a conflict between the activity of taping and the visual stimuli. This result indicated that when re-designing a product to stimulate novelty through aesthetic product experiences, the new added function is degraded when the aesthetic interaction conflicts with the product's practical function. However, the current study also indicated, through results pertaining to the Type $C$ (Auditory) design, how particular visceral stimuli, in this case visual, misaligned with functional requirements to negatively implicate the potential benefit in terms the aesthetic product experience.

Related to the above, Type $C$ Auditory (Figure 6 red bars) received the highest mean $(\bar{x})$ response across the 7 positive response items compared to both Type A and Type B (Figure 7, red bars). In particular, Type $C$ (Auditory) received increased mean responses across the positive response items joy $(\bar{x}=3.24)$, 
fascination $(\bar{x}=3.19)$ and admiration $(\bar{x}=3.13)$. This result indicated the auditory aesthetic intervention (playing the 'Happy Birthday' tune while in use) was most successful in stimulating positive emotional response during the product experience compared to the other two designs. This result was supported by qualitative participant responses at interview. For example, Participant 01 suggested, "It is the most beautiful tape dispenser I've seen in my life", while Participant 02 mentioned, "I usually forget where the tape dispenser is in my office. But, it seems to have some interesting elements so that I want to keep it around". These qualitative results appeared to support those of the PrEmo study in suggesting the Type $C$ (Auditory) intervention as most effective in providing an enhanced product experience. We speculate that the auditory stimulation this result was derived from the fact that the aesthetic intervention did not impede the functional objectives of the product's use (i.e. wrapping a present). That is, the novelty of listening to the 'Happy Birthday' tune while wrapping did not obstruct a necessity to achieve the goal task.

\section{Discussion}

The current study adopted a research through design (Frens, 2007) approach to the design, development and prototyping of three products (tape-dispensers) for use in understanding how the application of visceral stimuli (visual and auditory) may implicate the product/user experience. A study was run for this research purpose and participants asked to use the product stimuli to wrap a gift. Resulting data was then analysed to understand how the introduction of different sensorial stimulus (within three different dispenser designs) may implicate the product experience.

Unsurprisingly, the introduction of both visual (Type B design, short animation) and auditory (Type $C$ design, 'Happy Birthday' tune) resulted in participants' more positive self-report towards the product experience. In particular, the Type $C$ (auditory) design was assessed more positively through 7 positive emotional response items. Type B design (Visual) received the next highest score, with Type A (Control) the lowest. This result indicated how stimulation of visual and auditory visceral response (Norman, 2004) appeared to lead to more positive product experience (as indicated by more positive self-report scores).

More interestingly, however, results also indicated the Type $C$ (Auditory) design as providing the most positive product experience among the three designs. The further analysis of qualitative data, gathered during interview and think-aloud sessions with the same participants, indicated how positive emotions towards aesthetic interaction may have been dependent upon the profiles of individual participants themselves, as well as the tape dispensers' ability to achieve the level of product functionality expected. That is, the introduction of the auditor aesthetic stimuli (i.e. 'Happy Birthday' tune) did not negatively impact a functional requirement to wrap the gift.

In agreement with the literature in consumer profiles (Batra and Ahtola, 1990), the participant group appeared to contain individuals that were more inclined towards aesthetic product experiences, and their resulting stimulation of emotion compared to others. For example, when comparing responses towards Type B (visual) and Type A (Control) designs, some subjects appears to express disappointment with the products lack of novelty, "I prefer Type B rather than Type A because this tape dispenser so far is not funny and is only for the function of disconnecting the tape". In contrast, others spoke of a necessity for functionality as core to any evaluation of the product experience, "I prefer Type A more than B because $B$ is functionally difficulty to use and strange for me". This result indicated that, while stimulation of emotion through visual stimuli offered increased opportunity for positive product experience, this depended upon the particular profiles of users who, on the one hand, may be more inclined to value novel approaches, while others may respond more negatively due to increased concern over functional requirements of product use.

Related to the above, the Type $C$ (Auditory) design was also evaluated more positively then the visual animation intervention (Type B, Visual). Results indicated how the animation may have distracted from the practical necessity of the product's use (i.e. wrapping up the present). In contrast, the auditory stimulation provided by the Type $C$ design, had no such interference; wrapping a gift makes limited use of hearing compared to sight. This result was supported by qualitative participant responses, indicating how engagement with the animation may have frustrated the functional requirement of supporting the gift wrapping activity, "Type B is also a new approach, but it was hard to see it [animation] while 
cutting the tape. Type C, however, was easier to use and fun because it sounds natural" (Participant 12).

Results thus indicated how the application of features to stimulate positive emotional response through novel aesthetic interactions during product experience requires careful consideration for how aesthetic features may relate to and support product function and practical necessity (in this case the functionality of a tape dispenser in wrapping a gift). To this end, it would be interesting to explore how the application of auditory and visual elements may implicate the product experience when applied to other products (i.e. juicer, toothbrush, comb etc.). It would also be interesting to study how stimuli applied to the same product, but used to achieve different practical goals may implicate the product experience (i.e. a tape dispenser used for different purposes).

In addition, the current study indicates how visceral stimuli may change the users' emotion positively, leading to a novel experience. Both stimuli (Type $C$ auditory and Type $B$ visual) succeeded in altering the participants' emotional state. However, can it be said that this led to design-driven innovation in meaning change as first proposed by Verganti (2008)? As mentioned in our introduction, the concept of design-driven innovation is founded upon a notion of radical change in the meaning of a product experience. Our results have indicated how some participants interpreted the meaning of the product experience differently from the conventional product archetype (Type A control). Results also indicated how auditory stimuli changed the meaning of a gift wrapping experience for some. That is, they responded to the tape dispenser with auditory stimuli as a more playful product compared to the utility of a standard dispenser. However, findings have also indicated that, in order to stimulate the positive meaning change discussed by Verganti $(2003,2008)$, aesthetic product experiences may work as a catalyst for meaning change, but only where novel, emotional experiences do not conflict with practical necessity. Moreover, meaning, as proposed by both Verganti (ibid) and others (Norman, 2004; Desmet and Hekkert, 2007) rests upon a notion that the user own interpretation of the meaning of a product experiences, rests upon the values, experiences and expectations they themselves bring to the experience. Further studies may wish to explore how meaning is implicated by previous product experience, with particular focus upon functional expectations and response to novel aesthetic interventions. Finally, and related to the above, results also indicated how response to potentially novel product experiences, driven by the application of aesthetic interactions, is dependent upon satisfying expectations related to product functionality, together with the user's sensitivity towards the product's fulfilment of practical requirements.

\section{Conclusions}

Although the current study has provided an indication of how aesthetic product attributes may be applied to drive more novel product experiences, we also see limitations in our approach. First, the product chosen was a relatively simple, low-fidelity tape dispenser. It is unclear how novel stimuli applied to other products and product categories would implicate emotional response. Further, the current study did not well control for or measure the influence of individual differences between participants. That is, how might the experiences, attitudes and characteristics participants bring to the product experience implicate response to novel visceral stimuli during the user/product experience? Finally, the approach did not control for the assigned task of gift wrapping. That is, how may the activity of wrapping a present have implicated emotional response? Further studies applying the products to different tasks and contexts are required to better understand their role as driver for emotional response. Moreover, the current study adopted aesthetic interaction as framework for understanding emotional response during user product experiences. However, future studies may wish to explore the influence of novel product features through other lenses; affective engineering or the Kansei approach for example. Finally, in the current study we have attempted to type aesthetic product experiences and meaning innovation through the pursuit of novelty they both share. However, further work is required to both better define meaning innovation, as construct to understand product innovation and its relation to novel product experiences. These limitations notwithstanding, the current study has indicated the importance of consistency between core product function and any novel stimuli. If aesthetic product experiences is to be used as means to drive meaning innovation as described by Verganti (2008), more work is now required. For example, it would be interesting for further studies to explore how different user profiles may respond 
to the same visual stimuli and implications for the product experience. Also, future works might examine how embedding the animation and/or birthday tune into different products and their associated different tasks may implicate user responses.

\section{Acknowledgements}

This work was supported by the 'Promotion of Graduate School of Creative Design Engineering' of the Korea Institute of Design Promotion with a grant from the Ministry of the Trade, Industry and Energy, Republic of Korea. (N0001436)

\section{References}

American Chemical Society (2017), Scotch Transparent Tape - National Historic Chemical Landmark. [online] Available at: https://www.acs.org/content/acs/en/education/whatischemistry/landmarks/scotchtape.html (accessed 03.07.2017).

Batra, R. and Ahtola, O. (1990), "Measuring the Hedonic and Utilitarian Sources of Consumer Attitudes", Marketing Letters, Vol. 2 No. 2, pp. 159-170.

Bergghman, M. and Hekkert, P. (2016), “The Beauty of Balance: An empirical integration of the Unified Model of Aesthetics for Product Design", Proceedings of DRS2016, Brighton, UK.

Demir, E., Desmet, P.M.A. and Hekkert, P. (2009), "Appraisal Patterns of Emotions in Human-Product Interaction", International Journal of Design, Vol. 3 No. 2, pp. 41-51.

Desmet, P. and Hekkert, P. (2007), "Framework of product experience", International Journal of Design, Vol. 1 No. 1, pp. 57-66.

Desmet, P., Nicolás, J.C.O. and Schoormans, J.P. (2008), "Product Personality in Physical Interaction", Design Studies, Vol. 29 No. 5, pp. 458-477.

Desmet, P. and Schifferstein, H. (2012), "Emotion Research as Input for Product Design”, In: Beckley, J., Paredes, D. and Lopetcharats, K. (Eds.), Product Innovation Toolbox: A Field Guide to Consumer Understanding and Research, John Wiley and Sons, pp. 149-175. https://doi.org/10.1002/9781118229248.ch6e

Frens, J. (2007), "Research through Design: a Camera Case Study", In: Michel, R. (Ed.), Design Research Now: Essays and Selected Projects, Birkhäuser Basel, Basel, pp. 135-154.

Hallnas, L. (2011), "On the Foundations of Interaction Design Aesthetics: Revisiting the Notion of Form and Expression”, International Journal of Design, Vol. 5 No. 1, pp. 73-84.

Hekkert, P., Snelders, D. and Van Wieringen, P. (2003), "'Most advanced, yet acceptable': Typicality and novelty as joint predictors of aesthetic preference in industrial design", British Journal of Psychology, Vol. 94 No. 1, pp. 111-124.

Hung, W.K. and Chen, L.L. (2012), "Effects of novelty and its dimensions on aesthetic preference in product design", International Journal of Design, Vol. 6 No. 2, pp. 81-90.

Locher, P., Overbeeke, K. and Wensveen, S. (2010), “Aesthetic Interaction: A Framework", Design Issues, Vol. 26 No. 2, pp. 70-79. https://doi.org/10.1162/DESI_a_00017

Yasukuni, M. (2011), Notchless: Adhesive- Tape Dispenser. [online] Available at: http://notchless.com/english/index.html (accessed 12.08.2017).

M-S-D-S Studio (2015), Tape Dispencer. [online] Available at: http://msds-studio.ca/projects/tape-dispenser (accessed 15.12.2017).

Norman, D. (2002), Knowing What To Do. The Design of Everyday Things, 2nd ed., Basic Books, New York.

Norman, D. (2004), Emotional Design: Why we love (or hate) everyday things, Basic Books, New York.

Pahk, Y. and Self, J. (2015), "Knowledge of use and acceptability of typological innovation: A comparative study", Proceedings of IASDR 2015, Brisbane, Australia.

Rampino, L. (2011), "The Innovation Pyramid: A Catagorization of the Innovation Phenomenon in the Productdesign Field”, International Journal of Design, Vol. 5 No. 1, pp. 3-16.

Reilink, D. (2017), Meet ClickTape. [online] Available at: http://derkreilink.com/ (accessed 11.06.2017).

SusanGroup (2017), PrEmo. [online] Available at: http://www.premotool.com/ (accessed 27.10.2017).

Strauss, A. and Corbin, J. (1994), "Grounded Theory Methodology - An Overview”, In: Norman, K.D. and Vannaeds, S.L.Y. (Eds.), Handbook of qualitative research, Sage Publications, Thousands Oaks, pp. $273-285$.

Tractinsky, N. (2005), "Does Aesthetics Matter in Human-Computer Interaction?”, In: Stary, C. (Ed.), Mensch and Computer, Oldenbourg Verlag, München, pp. 29-42.

Udsen, L.E. and Jørgensen, A.H. (2005), "The Aesthetic Turn: unravelling recent aesthetic approaches to humancomputer interaction", Digital Creativity, Vol. 16 No. 4, pp. 205-216. https://doi.org/10.1080/14626260500476564 
Verganti, R. (2003), “Design as brokering of languages: Innovation strategies in Italian rms”, Design Management Journal, Vol. 14 No. 3, pp. 34-46.

Verganti, R. (2008), "Design, Meanings, and Radical Innovation: A Metamodel and a Research Agenda", Journal of Product Innovation Management, Vol. 25 No. 5, pp. 436-456. https://doi.org/10.1111/j.15405885.2008.00313.x.

Verganti, R. (2009), Design-driven Innovation: Changing the Rules of Competition by Radically Innovating What Things Mean, Harvard Business Press, Boston.

Zimmerman, J., Forlizzi, J. and Evenson, S. (2007), "Research through design as a method for interaction design research", Proceedings of CHI / Conference on Human Factors in Computing Systems, San Jose, CA, USA, ACM Press, New York, USA, pp. 493-502.

Kwangyoung Lee, Master candidate

Ulsan National Institute of Science and Technology, Creative Design Engineering

50, UNIST-gil, Ulsan 44919, Republic of Korea, 44919 Ulsan, South Korea

Email: dlight@unist.ac.kr 